\title{
Nonlocal-derivative NLS equations and group-invariant soliton solutions
}

Yuqin Yao ${ }^{1 *}$ and Yehui Huang ${ }^{2}$

"Correspondence: yyqinw@126.com 1 Department of Applied Mathematics, China Agricultural University, Beijing, China Full list of author information is available at the end of the article

\begin{abstract}
A coupled Chen-Lee-Liu (CLL) system is proposed and its linear Lax pair is given. Many kinds of nonlocal-derivative NLS (DNLS) equations arise from the group symmetry reductions of the coupled CLL system. $\hat{P} \hat{T} \hat{C}$-symmetry invariant one-soliton solution and periodic two-soliton solution of a two-place DNLS (TDNLS) system are obtained. A group symmetry invariant two-soliton solution of a four-place DNLS (FDNLS) system is worked out. New characteristics of the two-soliton interactions for the TDNLS system and FDNLS system are analyzed.
\end{abstract}

Keywords: Nonlocal-derivative NLS equation; Bilinear method; Exact solutions; Soliton

\section{Introduction}

It is well known that many physical problems may occur in two or more places which are linked to each other, which can be called multi-place problem. To describe two-place problems, Alice-Bob systems (ABs) [1] are proposed. That is, if $A(x, t)$ is Alice's state and $B\left(x^{\prime}, t^{\prime}\right)$ is Bob's state, there is a suitable operator $\hat{f}$ which can linked to the two states at the same time,

$$
B\left(x^{\prime}, t^{\prime}\right)=\hat{f} A(x, t)=A^{\hat{f}}, \quad A(x, t)=\hat{f}^{-1} B\left(x^{\prime}, t^{\prime}\right)=B^{\hat{f}^{-1}} .
$$

The equivalence assumption requires that the operator $\hat{f}$ satisfies $\hat{f}^{2}=1$. Usually, $\left(x^{\prime}, t^{\prime}\right)$ is far from $(x, t)$. Hence, the two-place systems or Alice-Bob systems (ABs) are nonlocal. When the operator $\hat{f}$ is taken as a special case, many kinds of nonlocal integrable systems can be obtained. For example, this nonlocal nonlinear Schrödinger (NLS) equation is proposed by Ablowitz and Musslimani [2]:

$$
\begin{aligned}
& i A_{t}+A_{x x} \pm A^{2} B=0, \\
& B=\hat{f} A=\hat{P} \hat{C} A=A^{*}(-x, t),
\end{aligned}
$$

with $\hat{f}=\hat{P} \hat{C}$, where $\hat{P}$ and $\hat{C}$ are the parity and charge conjugation operators, respectively, $*$ is for the complex conjugate. Recently, the nonlocal NLS equation was derived in a physical application of magnetics [3]. Excited by the pioneering work, the nonlocal integrable

(c) The Author(s) 2020. This article is licensed under a Creative Commons Attribution 4.0 International License, which permits use, sharing, adaptation, distribution and reproduction in any medium or format, as long as you give appropriate credit to the original author(s) and the source, provide a link to the Creative Commons licence, and indicate if changes were made. The images or other third party material in this article are included in the article's Creative Commons licence, unless indicated otherwise in a credit line to the material. If material is not included in the article's Creative Commons licence and your intended use is not permitted by statutory regulation or exceeds the permitted use, you will need to obtain permission directly from the copyright holder. To view a copy of this licence, visit http://creativecommons.org/licenses/by/4.0/. 
systems have attracted considerable attention in recent years. At present, the nonlocal $\mathrm{KdV}$ equation $[4,5]$, the nonlocal $\mathrm{mKdV}$ equation [6-8], the nonlocal discrete NLS equation [9], the nonlocal KP equation [10,11], the nonlocal DS equation [12-14], and so on [15-17] have been studied.

Solitons represent robust nonlinear coherent structures and have been theoretically studied and observed in experiments in physical, chemical and biological science [18-20]. At present, many methods [21-40] have been developed to search for solitons of nonlinear evolution equations. Among them, the function expansion method [21-25], the bilinear method [33, 34], Darboux transformation [35, 36], the symmetry reduction method $[37,38]$ and the Riemann-Hilbert approach $[39,40]$ are very effective and widely used methods. For example, in [22], soliton solutions for a type of mKdV equation with a first local-derivative term are obtained based on the Riccati-Bernoulli sub-ordinary differential equation and a modified tanh-coth method. New solitary solutions for the ZakharovKuznetsov equation are worked out by a generalized exponential rational function method in [23]. The dark, bright, dark-bright, dark-singular and singular soliton of the NLS equation with quadratic-cubic nonlinearity are derived by adopting the sine-Gordon expansion method in [24]. The exact traveling wave solutions for the fractional equations and the heat transfer equations are worked out in Refs. [41-48].

With the advent of the nonlocal systems, the methods mentioned above have been developed to construct the soliton solutions of the nonlocal systems [49-57]. Meanwhile, there is few work about the soliton solutions of the nonlocal four-place systems. In this paper, new nonlocal two-place DNLS (TDNLS) and four-place DNLS (FDNLS) systems are derived based on the coupled Chen-Lee-Liu (CLL) system and the $\hat{P} \hat{T} \hat{C}$-symmetry group. A linear Lax pair is given which guarantees the integrability of the nonlocal TDNLS system and FDNLS system. In order to construct the group-invariant soliton solutions, we first rewrite the solutions of the DNLS equation [58] in the form expressed by hyperbolic and triangular functions. Then the $\hat{P} \hat{T} \hat{C}$-symmetry invariant one-soliton solution and periodic two-soliton solution of a new TDNLS system are obtained. Further, we also work out the group-invariant two-soliton solution of a FDNLS system. There is some interesting dynamics appearing in the TDNLS system and FDNLS system, different from the dynamics of the local DNLS equation.

The paper is organized as follows. In Sect. 2, we construct the coupled CLL system and its Lax pair is given. Some new nonlocal TDNLS system and FDNLS system arise from the group symmetry reductions of the coupled CLL system. In Sect. 3, the expressions of group-invariant soliton solutions for the nonlocal DNLS system are presented and the multi-soliton solutions of the TDNLS system and the FDNLS system are worked out. A conclusion is given in the last section.

\section{Nonlocal multi-place derivative NLS system}

The derivative NLS (DNLS) equation

$$
i q_{t}+q_{x x}+2 i q q^{*} q_{x}=0
$$

can be reduced from the Chen-Lee-Liu (CLL) system [59]

$$
\begin{aligned}
& q_{t}=q_{x x}+2 q r q_{x}, \\
& r_{t}=-r_{x x}+2 q r r_{x},
\end{aligned}
$$


by setting $r=q^{*}$ and replacing $t$ by $i t$ and $x$ by $-i x$. From the coupled system (4), some different kinds of nonlocal integrable DNLS equations can be obtained by using the $\hat{P} \hat{T} \hat{C}$ symmetry reductions. In the following, we first find some kinds of integrable coupled CLL systems. Here is the first non-trivial coupled CLL system

$$
\begin{aligned}
& q_{t}=q_{x x}+2(p+q)^{2}(r+s)(p r-q s)+2 q(r+s)\left(p_{x}+q_{x}\right)+2[(p+q)(q s-p r)]_{x}, \\
& p_{t}=p_{x x}-2(p+q)^{2}(r+s)(p r-q s)+2 p(r+s)\left(p_{x}+q_{x}\right)+2[(p+q)(p r-q s)]_{x}, \\
& r_{t}=-r_{x x}+2(p+q)(r+s)^{2}(p r-q s)+2 r(p+q)\left(r_{x}+s_{x}\right)+2[(r+s)(p r-q s)]_{x} \\
& s_{t}=-s_{x x}-2(p+q)(r+s)^{2}(p r-q s)+2 s(p+q)\left(r_{x}+s_{x}\right)+2[(r+s)(q s-p r)]_{x} .
\end{aligned}
$$

It is obvious that the coupled CLL system (5) can be reduced to the standard CLL system if we take $p=q$ and $r=s$. The integrability of the coupled CLL system (5) can be guaranteed by the following Lax pair:

$$
\begin{aligned}
& \Psi_{x}=M \Psi, \\
& \Psi_{t}=N \Psi, \quad \Psi=\left(\psi_{1}, \psi_{2}\right)^{T},
\end{aligned}
$$

with

$$
\begin{aligned}
& M=\left[\begin{array}{cccc}
-\frac{1}{2}\left(\lambda^{2}-(p+q)(r+s)\right) & (q+q) \lambda & 0 & 0 \\
(r+s) \lambda & \frac{1}{2}\left(\lambda^{2}-(p+q)(r+s)\right) & 0 & 0 \\
0 & (p-q) \lambda & -\frac{1}{2}\left(\lambda^{2}-(p+q)(r+s)\right) & (q+q) \lambda \\
(r-s) \lambda & 0 & (r+s) \lambda & \frac{1}{2}\left(\lambda^{2}-(p+q)(r+s)\right)
\end{array}\right], \\
& N=\left[\begin{array}{cccc}
n_{11} & n_{12} & 0 & 0 \\
n_{21} & -n_{11} & 0 & 0 \\
n_{31} & n_{32} & n_{11} & n_{12} \\
n_{41} & -n_{31} & n_{21} & -n_{11}
\end{array}\right] \text {, }
\end{aligned}
$$

where

$$
\begin{aligned}
& n_{11}=\alpha \lambda^{4}+(p+q)(r+s) \lambda^{2}-\frac{1}{2}(p+q)^{2}(r+s)^{2}-\frac{1}{2}(r+s)\left(p_{x}+q_{x}\right)+\frac{1}{2}(p+q)\left(r_{x}+s_{x}\right), \\
& n_{12}=(p+q) \lambda^{3}+\left[-(p+q)_{x}-(p+q)^{2}(r+s)\right] \lambda \\
& n_{21}=(r+s) \lambda^{3}+\left[(r+s)_{x}-(p+q)(r+s)^{2}\right] \lambda \\
& n_{31}=\beta \lambda^{4}+2(p r-q s) \lambda^{2}+8(p+q)(r+s)(q s-p r)-2 r p_{x}+2 s q_{x}+2 p r_{x}-2 q s_{x} \\
& n_{41}=(r-s) \lambda^{3}+\left[r_{x}-s_{x}-4(r+s)(p r-q s)-(p+q)(r+s)(r-s)\right] \lambda .
\end{aligned}
$$

The full $\hat{P} \hat{T} \hat{C}$-symmetry group $\Theta$ possesses the form [10]

$$
\Theta=\{1, \hat{P}, \hat{T} \hat{C}, \hat{P} \hat{T} \hat{C}\} \cup \hat{C}\{1, \hat{P}, \hat{T} \hat{C}, \hat{P} \hat{T} \hat{C}\}=\Theta_{1} \cup \Theta_{1}^{C}
$$


Using the sub-symmetry group $\Theta_{1}$ and the symmetry coset $\Theta_{1}^{C}$, we obtain two kinds of symmetry reductions from the coupled CLL system (5)

$$
\begin{aligned}
q_{t}= & q_{x x}+2\left(q^{\hat{f}_{k}}+q\right)^{2}\left(r+r^{\hat{f}_{k}}\right)\left(q^{\hat{f}_{k}} r-q r^{\hat{f}_{k}}\right)+2 q\left(r+r^{\hat{f}_{k}}\right)\left(q^{\hat{f}_{k}}+q\right)_{x} \\
& +2\left[\left(q^{\hat{f}_{k}}+q\right)\left(q \hat{r}^{\hat{f}_{k}}-q^{\hat{f}_{k}} r\right)\right]_{x}, \\
r_{t}= & -r_{x x}+2\left(q^{\hat{f}_{k}}+q\right)\left(r+r^{\hat{f}_{k}}\right)^{2}\left(q^{\hat{f}_{k}} r-q r^{\hat{f}_{k}}\right)+2 r\left(q^{\hat{f}_{k}}+q\right)\left(r+r^{\hat{f}_{k}}\right)_{x} \\
& +2\left[\left(r+r^{\hat{f}_{k}}\right)\left(q^{\hat{f}_{k}} r-q r^{\hat{f}_{k}}\right)\right]_{x}, \\
\hat{f}_{k} \in & \Theta_{1}=\{1, \hat{P}, \hat{T} \hat{C}, \hat{P} \hat{T} \hat{C}\}, \quad(p, s)=\hat{f}_{k}(q, r),
\end{aligned}
$$

and

$$
\begin{aligned}
q_{t}= & q_{x x}+2(p+q)^{2}\left(q^{\hat{g_{j}}}+p^{\hat{g_{j}}}\right)\left(p q^{\hat{g}_{j}}-q p^{\hat{g_{j}}}\right)+2 q\left(q^{\hat{g_{j}}}+p^{\hat{\hat{g}_{j}}}\right)\left(p_{x}+q_{x}\right) \\
& +2\left[(p+q)\left(q p^{\hat{g_{j}}}-p q^{\hat{g}_{j}}\right)\right]_{x}, \\
p_{t}= & p_{x x}-2(p+q)^{2}\left(q^{\hat{g_{j}}}+p^{\hat{g_{j}}}\right)\left(p q^{\hat{g}_{j}}-q p^{\hat{g}_{j}}\right)+2 p\left(q^{\hat{g_{j}}}+p^{\hat{g}_{j}}\right)\left(p_{x}+q_{x}\right) \\
& +2\left[(p+q)\left(p q^{\hat{g}_{j}}-q p^{\hat{g}_{j}}\right)\right]_{x^{\prime}} \\
\hat{g}_{j} \in & \Theta_{1}^{C}=\{\hat{C}, \hat{T}, \hat{C} \hat{P}, \hat{P} \hat{T}\}, \quad(r, s)=\hat{g}_{j}(q, p),
\end{aligned}
$$

respectively. Furthermore, based on the systems (7) and (8), we can work out 16 different types of DNLS systems

$$
\begin{aligned}
& q_{t}=q_{x x}+2\left(q^{\hat{f}_{k}}+q\right)^{2}\left(q^{\hat{g}_{j}}+q^{\hat{f}_{k} \hat{g}_{j}}\right)\left(q^{\hat{f}_{k}} q^{\hat{g}_{j}}-q q^{\hat{f}_{k} \hat{g}_{j}}\right) \\
& \quad+2 q\left(q^{\hat{g}_{j}}+q^{\hat{f}_{k} \hat{g}_{j}}\right)\left(q^{\hat{f}_{k}}+q\right)_{x}+2\left[\left(q^{\hat{f}_{k}}+q\right)\left(q q^{\hat{f_{k}} \hat{g}_{j}}-q^{\hat{f}_{k}} q^{\hat{g}_{j}}\right)\right]_{x^{\prime}} \\
& (p, r, s)=\left(q^{\hat{f}_{k}}, q^{\hat{g}_{j}}, q^{\hat{f}_{k} \hat{g}_{j}}\right), \\
& \hat{f}_{k} \in \Theta_{1}=\{1, \hat{P}, \hat{T} \hat{C}, \hat{P} \hat{T} \hat{C}\}, \hat{g}_{j} \in \Theta_{1}^{C}=\{\hat{C}, \hat{T}, \hat{C} \hat{P}, \hat{P} \hat{T}\} .
\end{aligned}
$$

For example, if we take $\hat{f}_{k}=1, \hat{g}_{j}=\hat{C}$ in (9), the local DNLS equation is given by

$$
q_{t}=q_{x x}+8 q q^{*} q_{x}
$$

When we take $\hat{f}_{k}=1, \hat{g}_{j}=\{\hat{T}, \hat{P} \hat{C}, \hat{P} \hat{T}\}$ or $\hat{g}_{j}=\hat{C}, \hat{f}_{k}=\{\hat{P}, \hat{T} \hat{C}, \hat{P} \hat{T} \hat{C}\}$, a two-place nonlocal DNLS systems can be obtained:

$$
\begin{aligned}
& q_{t}=q_{x x}+2(p+q)^{2}(r+s)(p r-q s)+2 q(r+s)\left(p_{x}+q_{x}\right)+2[(p+q)(q s-p r)]_{x^{\prime}} \\
& (p, r, s)=\left(q^{\hat{f}_{k}}, \quad q^{\hat{g}_{j}}, q^{\hat{f}_{k} \hat{g}_{j}}\right) .
\end{aligned}
$$

For instance, for $\hat{f}_{k}=1, \hat{g}_{j}=\hat{P} \hat{C}$, Eq. (10) becomes

$$
q_{t}=q_{x x}+8 q q^{*}(-x, t) q_{x}
$$


For $\hat{f}_{k}=\hat{P} \hat{T} \hat{C}, \hat{g}_{j}=\hat{C}$, Eq. (10) becomes

$$
\begin{aligned}
q_{t}= & q_{x x}+2\left[q+q^{*}(-x,-t)\right]^{2}\left[q^{*}+q(-x,-t)\right]\left[q^{*} q^{*}(-x,-t)-q q(-x,-t)\right] \\
& +2 q\left[q^{*}+q(-x,-t)\right]\left[q+q^{*}(-x,-t)\right]_{x} \\
& +2\left[\left(q+q^{*}(-x,-t)\right)\left(q q(-x,-t)-q^{*} q^{*}(-x,-t)\right)\right]_{x} .
\end{aligned}
$$

The systems (10) to (12) are all called two-place nonlocal DNLS equation.

If we take $\hat{f}_{k}=\hat{P}, \hat{g}_{j}=\{\hat{T}, \hat{P} \hat{T}\}, \hat{f}_{k}=\hat{T} \hat{C}, \hat{g}_{j}=\{\hat{C} \hat{P}, \hat{P} \hat{T}\}$ or $\hat{f}_{k}=\hat{P} \hat{T} \hat{C}, \hat{g}_{j}=\{\hat{T}, \hat{P} \hat{C}\}$, some four-place nonlocal DNLS equations can be obtained:

$$
\begin{aligned}
& q_{t}=q_{x x}+2(p+q)^{2}(r+s)(p r-q s)+2 q(r+s)\left(p_{x}+q_{x}\right)+2[(p+q)(q s-p r)]_{x} \\
& (p, r, s)=\left(q^{\hat{f}_{k}}, q^{\hat{g}_{j}}, q^{\hat{f}_{k} \hat{g}_{j}}\right), \\
& \left(\hat{f}_{k}, \hat{g}_{j}\right)=(\hat{P}, \hat{T}(1, \hat{P})),(\hat{T} \hat{C}, \hat{P}(\hat{C}, \hat{T})),(\hat{P} \hat{T} \hat{C},(\hat{T}, \hat{P} \hat{C})) .
\end{aligned}
$$

For example, for $\hat{f}_{k}=\hat{T} \hat{C}, \hat{g}_{j}=\hat{P} \hat{C}$, Eq. (13) becomes

$$
\begin{aligned}
q_{t}= & q_{x x}+2\left[q+q^{*}(x,-t)\right]^{2}\left[q(-x, t)+q^{*}(-x, t)\right]\left[q^{*}(-x, t) q^{*}(x,-t)-q q(-x,-t)\right] \\
& +2 q\left[q^{*}(-x, t)+q(-x,-t)\right]\left[q^{*}(x,-t)+q\right]_{x} \\
& +2\left[\left(q+q^{*}(x,-t)\right)\left(q q(-x,-t)-q^{*}(x,-t) q^{*}(-x, t)\right)\right]_{x} .
\end{aligned}
$$

For $\hat{f}_{k}=\hat{P} \hat{T} \hat{C}, \hat{g}_{j}=\hat{P} \hat{C}$, Eq. (13) becomes

$$
\begin{aligned}
q_{t}= & q_{x x}+2\left[q+q^{*}(-x,-t)\right]^{2}\left[q(x,-t)+q^{*}(-x, t)\right]\left[q^{*}(-x,-t) q^{*}(-x, t)-q q(x,-t)\right] \\
& +2 q\left[q^{*}(-x, t)+q(x,-t)\right]\left[q^{*}(-x,-t)+q\right]_{x} \\
& +2\left[\left(q+q^{*}(-x,-t)\right)\left(q q(x,-t)-q^{*}(-x,-t) q^{*}(-x, t)\right)\right]_{x} .
\end{aligned}
$$

Equations (13) to (15) are all four-place nonlocal DNLS equations.

\section{$3 \hat{P} \hat{T} \hat{C}$-invariant multi-soliton solutions of the DNLS type multi-place system}

In Ref. [58], the bilinear form of the generalized DNLS equation

$$
\begin{aligned}
& q_{t}=q_{x x}+2 q r q_{x}, \\
& r_{t}=-r_{x x}+2 q r r_{x},
\end{aligned}
$$

is worked out and

$$
\begin{aligned}
& \left(D_{t}-D_{x}^{2}\right) g \cdot f=0, \\
& \left(D_{t}+D_{x}^{2}\right) h \cdot s=0, \\
& D_{x}^{2} f \cdot s=i D_{x} g \cdot h, \\
& D_{x} f \cdot s=g h,
\end{aligned}
$$


by taking the variable transformation $q=\frac{g}{f}, r=\frac{h}{s}$ and making use of some identities. As a case of reduction, taking $r=q^{*}$, i.e. $s=f^{*}, h=g^{*}$ and replacing $t$ by it and $x$ by $-i x$, the generalized DNLS equation (16) reduces to the DNLS equation (3) and the bilinear equation (17) reduces to

$$
\begin{aligned}
& \left(i D_{t}+D_{x}^{2}\right) g \cdot f=0, \\
& D_{x}^{2} f \cdot f^{*}=i D_{x} g \cdot g^{*}, \\
& D_{x} f \cdot f^{*}=i g g^{*} .
\end{aligned}
$$

Equation (18) just is the bilinear equation of the the DNLS equation (3).

Its N-soliton solutions can be uniformly written as

$$
\begin{aligned}
& g_{n}=\sum_{\mu=0,1} A_{2}(\mu) \exp \left[\sum_{j=1}^{2 n} \mu_{j} \xi_{j}^{\prime}+\sum_{1 \leq j<\rho}^{2 n} \mu_{j} \mu_{\rho} \theta_{j \rho}\right], \\
& f_{n}=\sum_{\mu=0,1} A_{1}(\mu) \exp \left[\sum_{j=1}^{2 n} \mu_{j} \xi_{j}^{\prime \prime}+\sum_{1 \leq j<\rho}^{2 n} \mu_{j} \mu_{\rho} \theta_{j \rho}\right],
\end{aligned}
$$

where

$$
\begin{aligned}
& \xi_{j}=i k_{j} x-i k_{j}^{2} t+\xi_{j}^{(0)}, \quad \xi_{j}^{\prime}=\xi_{j}, \quad \xi_{n+j}^{\prime}=\xi_{j}^{*}+\log k_{j}^{*}, \\
& \xi_{j}^{\prime \prime}=\xi_{j}+\log k_{j}, \quad \xi_{n+j}^{\prime \prime}=\xi_{j}^{*} \quad(j=1,2, \ldots, n), \\
& e^{\theta_{j, n+\rho}}=\frac{1}{\left(k_{j}-k_{\rho}^{*}\right)^{2}} \quad(j, \rho=1,2, \ldots, n), \\
& e^{\theta_{j, \rho}}=\left(k_{j}-k_{\rho}\right)^{2}, \quad e^{\theta_{n+j, n+\rho}}=\left(k_{j}^{*}-k_{\rho}^{*}\right)^{2} \quad(j<\rho=2,3, \ldots, n),
\end{aligned}
$$

with arbitrary complex constants $\xi_{j}^{(0)}, j=1,2, \ldots, n$.

The summations $A_{1}(\mu)$ and $A_{2}(\mu)$ are taken over all possible combinations of $\mu_{j}=0,1$ $(j=1,2, \ldots, 2 n)$ and satisfy the following conditions:

$$
\sum_{j=1}^{n} \mu_{j}=\sum_{j=1}^{n} \mu_{n+j}, \quad \sum_{j=1}^{n} \mu_{j}=\sum_{j=1}^{n} \mu_{n+j}+1,
$$

respectively.

It is clear that the solution (19) is not $\hat{P} \hat{T} \hat{C}$-invariant for arbitrary $\xi_{j}^{(0)}$. So it is not the solution of the DNLS type multi-place system. In order to find $\hat{P} \hat{T} \hat{C}$-invariant solutions from (19), we rewrite $\xi_{j}$ as

$$
\begin{aligned}
\xi_{j} & =i k_{j} x-i k_{j}^{2} t+\eta_{0 j}-\frac{1}{2} \sum_{\rho=1}^{j-1} \theta_{\rho j}-\frac{1}{2} \sum_{\rho=j+1}^{2 n} \theta_{j \rho}-\frac{1}{2} \log k_{j} \\
& =\eta_{j}-\frac{1}{2} \sum_{\rho=1}^{j-1} \theta_{\rho j}-\frac{1}{2} \sum_{\rho=j+1}^{2 n} \theta_{j \rho}-\frac{1}{2} \log k_{j}, \\
\xi_{j}^{*} & =\eta_{j}^{*}-\frac{1}{2} \sum_{\rho=1}^{j-1} \theta_{\rho j}^{*}-\frac{1}{2} \sum_{\rho=j+1}^{2 n} \theta_{j \rho}^{*}-\frac{1}{2} \log k_{j}^{*} .
\end{aligned}
$$


We can prove that the solution (19) with (20) can be written as hyperbolic and triangular functions, which guarantees the $\hat{P} \hat{T} \hat{C}$-invariance when an appropriate constant is chosen. However, the general expression in terms of the hyperbolic and triangular functions is very complicated. So we only write down two examples for $n=1$ and $n=2$.

When $n=1$, the one-soliton solution for the DNLS equation (3) can be rewritten as

$$
q=\frac{\left(k_{1}-k_{1}^{*}\right) e^{\left(i \eta_{1 I}+\frac{1}{4} \log k_{1}^{*}-\frac{3}{4} \log k_{1}\right)}}{2 \cosh \left(\eta_{1 R}+\frac{1}{4} \log k_{1}-\frac{1}{4} \log k_{1}^{*}\right)} .
$$

When $n=2$, the two-soliton solution for the DNLS equation (3) can be rewritten as

$$
\begin{aligned}
& q=\left(| k _ { 1 } - k _ { 2 } | | k _ { 1 } - k _ { 2 } ^ { * } | \sqrt { k _ { 1 } ^ { * } k _ { 2 } ^ { * } } \left[\frac { k _ { 1 } - k _ { 1 } ^ { * } } { \sqrt { | k _ { 1 } | k _ { 1 } k _ { 2 } } } e ^ { i \eta _ { 1 I } } \operatorname { c o s h } \left[\eta_{2 R}+i(\alpha-\beta)\right.\right.\right. \\
& \left.+\frac{1}{4}\left(\log k_{2}^{*}-\log k_{2}\right)\right] \\
& \left.\left.+\frac{k_{2}-k_{2}^{*}}{\sqrt{\left|k_{2}\right| k_{1} k_{2}}} e^{i \eta_{2 I}} \cosh \left[\eta_{1 R}+i(\alpha+\beta)+\frac{1}{4}\left(\log k_{1}^{*}-\log k_{1}\right)\right]\right]\right) \\
& /\left(\left|k_{1}-k_{2}\right|^{2} \cosh \left(\eta_{1 R}+\eta_{2 R}+\frac{1}{4} \log \frac{k_{1} k_{2}}{k_{1}^{*} k_{2}^{*}}\right)\right. \\
& +\left|k_{1}-k_{2}^{*}\right|^{2} \cosh \left(\eta_{1 R}-\eta_{2 R}+\frac{1}{4} \log \frac{k_{1} k_{2}^{*}}{k_{1}^{*} k_{2}}\right) \\
& \left.-4 k_{1 I} k_{2 I} \cos \left(\eta_{1 I}-\eta_{2 I}-\frac{i}{4} \log \frac{k_{1} k_{1}^{*}}{k_{2} k_{2}^{*}}\right)\right) \text {. }
\end{aligned}
$$

Here

$$
\begin{aligned}
& k_{j}=k_{j R}+i k_{j I}, \quad j=1,2 \\
& \alpha=\arctan \frac{k_{1 I}-k_{2 I}}{k_{1 R}-k_{2 R}}, \quad \beta=\arctan \frac{k_{1 I}+k_{2 I}}{k_{1 R}-k_{2 R}},
\end{aligned}
$$

and $\eta_{j R}, \eta_{j I}$ are real and imaginary parts of $\eta_{j}$, respectively,

$$
\begin{aligned}
& \eta_{j R}=-k_{j I} x+2 k_{j R} k_{j I} t+\eta_{j 0 R}, \\
& \eta_{j I}=k_{j R} x-\left(k_{j R}^{2}-k_{j I}^{2}\right) t+\eta_{j 0 I},
\end{aligned}
$$

$\eta_{j 0 R}, \eta_{j 0 I}$ are arbitrary constants.

It is straightforward to test that (21) is $\hat{P} \hat{T} \hat{C}$-invariant for $\eta_{10 R}=0, \eta_{10 I}=$ $\frac{1}{2} \arccos \frac{k_{1}^{\frac{3}{2}}+\left(k_{1}^{\frac{3}{2}}\right)^{*}}{2\left(k_{1}-k_{1}^{*}\right)^{2} \sqrt{k_{1} k_{1}^{*}}}$. Equation (22) is $\hat{P} \hat{T} \hat{C}$-invariant for $\eta_{j 0 R}=\frac{1}{4} \log \frac{k_{j}}{k_{j}^{*}}, \quad \eta_{10 I}=$ $-\frac{i}{2} \log \frac{\sqrt{k_{j} k_{j}^{*}} k_{1} k_{2}}{\left(k_{j}-k_{j}^{*}\right)^{2}}(j=1,2)$ if $k_{j}+k_{j}^{*}=0$. As for the solution (22), we consider the reduction of $k_{1}=-k_{2}$. In the case of reduction, it can be tested that (22) is $\hat{P} \hat{T} \hat{C}$-invariant, $\hat{T} \hat{C}$-invariant and $\hat{P}$-invariant if $\eta_{j 0 R}=0, \eta_{j 0 I}=\frac{1}{2} \arccos \frac{i \sqrt{k_{j R}^{2}+k_{j I}^{2}} k_{j R}}{2 k_{j I}}(j=1,2)$.

\section{1 $\hat{P} \hat{T} \hat{C}$-invariant solutions of a nonlocal two-place DNLS equation}

In this section, we will give the $\hat{P} \hat{T} \hat{C}$-invariant multi-soliton solutions of a new nonlocal two-place DNLS equation. Replacing $t$ by it, $x$ by $-i x$ and $q$ by $\frac{1}{2} q$ in Eq. (12), we obtain 
the following nonlocal two-place DNLS equation:

$$
\begin{aligned}
i q_{t}+ & q_{x x}-\frac{1}{8}\left[q+q^{*}(-x,-t)\right]^{2}\left[q^{*}+q(-x,-t)\right]\left[q^{*} q^{*}(-x,-t)-q q(-x,-t)\right] \\
& +\frac{i}{2} q\left[q^{*}+q(-x,-t)\right] \\
& \times\left[q+q^{*}(-x,-t)\right]_{x}+\frac{i}{2}\left[\left(q+q^{*}(-x,-t)\right)\left(q q(-x,-t)-q^{*} q^{*}(-x,-t)\right)\right]_{x}=0 .
\end{aligned}
$$

Setting $q=q^{*}(-x,-t)$, Eq. (23) can be reduced to the DNLS equation (3), so

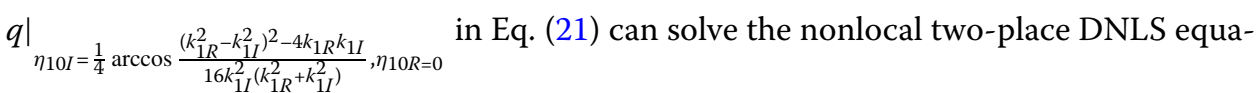
tion (23) with $\hat{f}=\hat{P} \hat{T}$. Thus Eq. (23) has the following one-soliton solution:

$$
q=\frac{e^{i\left(k_{1 R} x-\left(k_{1 R}^{2}-k_{1 I}^{2}\right) t\right)}}{2 \cosh \left(-k_{1 R} x+2 k_{1 R} k_{1 I} t+\frac{i}{2} \theta\right)}
$$

with

$$
\theta=\operatorname{arctant} \frac{k_{1 I}}{k_{1 R}} \text {. }
$$

This is a traveling wave at the speed of $2 k_{1 R} k_{1 I}$ with an initial phase $\frac{1}{2} \operatorname{arctant} \frac{k_{1 I}}{k_{1 R}}$. Figure 1 shows the shape and motion of the one-soliton case for $t=0$ and $t=1$.

When take $k_{j}=i k_{j I}(j=1,2)$ in Eq. (22), we can obtain the following two-soliton solutions of Eq. (23):

$$
\begin{aligned}
& q=\left(| k _ { 1 } - k _ { 2 } | | k _ { 1 } - k _ { 2 } ^ { * } | \sqrt { k _ { 1 } ^ { * } k _ { 2 } ^ { * } } \left[\frac{k_{1}-k_{1}^{*}}{\sqrt{\left|k_{1}\right| k_{1} k_{2}}} e^{i \eta_{1 I}} \cosh \left[\eta_{2 R}+i(\alpha-\beta)+\frac{1}{4}\left(\log k_{2}^{*}-\log k_{2}\right)\right]\right.\right. \\
& \left.\left.+\frac{k_{2}-k_{2}^{*}}{\sqrt{\left|k_{2}\right| k_{1} k_{2}}} e^{i \eta_{2 I}} \cosh \left[\eta_{1 R}+i(\alpha+\beta)+\frac{1}{4}\left(\log k_{1}^{*}-\log k_{1}\right)\right]\right]\right) \\
& /\left(\left|k_{1}-k_{2}\right|^{2} \cosh \left(\eta_{1 R}+\eta_{2 R}+\frac{1}{4} \log \frac{k_{1} k_{2}}{k_{1}^{*} k_{2}^{*}}\right)\right.
\end{aligned}
$$

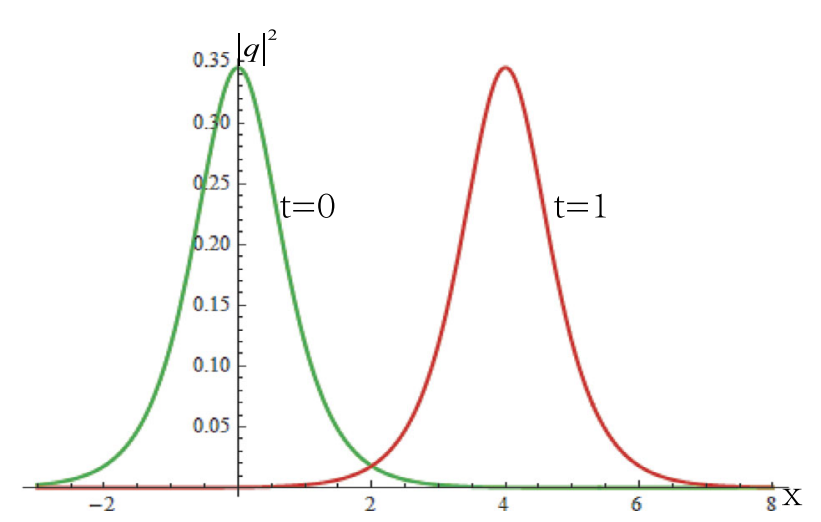

Figure 1 Motion of the one-soliton for the nonlocal two-place DNLS equation (23). The green one is the density distributions $|q|^{2}$ for $t=0$ and the red one is for $t=1$ with $k_{1 R}=1, k_{1 /}=2$ 


$$
\begin{aligned}
& +\left|k_{1}-k_{2}^{*}\right|^{2} \cosh \left(\eta_{1 R}-\eta_{2 R}+\frac{1}{4} \log \frac{k_{1} k_{2}^{*}}{k_{1}^{*} k_{2}}\right) \\
& \left.-4 k_{1 I} k_{2 I} \cos \left(\eta_{1 I}-\eta_{2 I}-\frac{i}{4} \log \frac{k_{1} k_{1}^{*}}{k_{2} k_{2}^{*}}\right)\right)
\end{aligned}
$$

with

$$
\eta_{j R}=-k_{j I} x, \quad \eta_{1 I}=k_{1 I}^{2} t+\frac{1}{2} \arccos \left(k_{2 I}\right), \quad \eta_{2 I}=k_{2 I}^{2} t+\frac{1}{2} \arccos \left(k_{1 I}\right),
$$

or

$$
\begin{aligned}
& \eta_{j R}=-k_{j I} x+\log \frac{k_{j}}{k_{j}^{*}} \quad(j=1,2), \\
& \eta_{1 I}=k_{1 I}^{2} t+\frac{1}{2} \arccos \left(k_{2 I}\right), \quad \eta_{2 I}=k_{2 I}^{2} t+\frac{1}{2} \arccos \left(k_{1 I}\right) .
\end{aligned}
$$

Figure 2 shows that the two-soliton solution is periodic with respect to time $t$ and localized in the $x$ direction. Figure 2(a) illustrates the density distributions $|q|^{2}$ on the $(x, t)$ plane and (b) expresses the interaction process of the two-soliton solution for $\eta_{j 0 R}=0$. Figure 2(c) shows the density distributions $|q|^{2}$ on $(x, t)$-plane and (d) describes the profiles of the two-soliton solution for different times when the constants $\eta_{j 0 R}=\log \frac{k_{j}}{k_{j}^{*}}$. It seems that the constant $\eta_{j 0 R}$ influences the interaction process of the two-soliton and the two solitons with the constants $\eta_{j 0 R}=\log \frac{k_{j}}{k_{j}^{*}}$ are apart from each other.

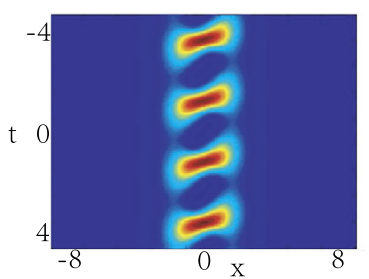

(a)

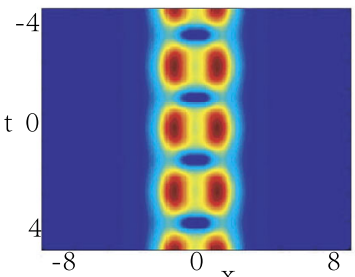

(c) ${ }^{\mathrm{x}}$

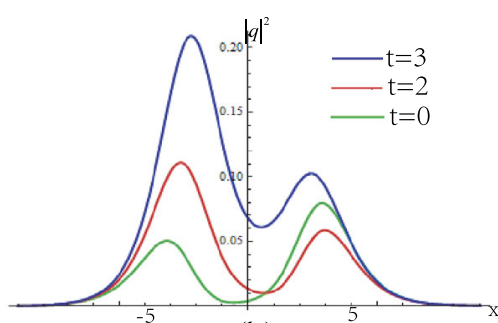

(b)

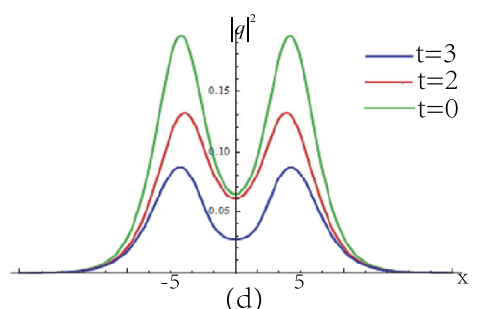

(d)

Figure 2 Interaction of the two-soliton solution for the nonlocal two-place DNLS equation (23). Pictures (a) and (b) show the interaction of the two-soliton for $\eta_{j 0 R}=0, \eta_{10 l}=\frac{1}{2} \arccos k_{2 l}, \eta_{20 l}=\frac{1}{2} \arccos k_{1 /}$. Pictures (c) and (d) illustrate the interaction of the two-soliton for $\eta_{j 0 R}=\log \frac{k_{j}}{k_{j}^{*}}, \eta_{10 l}=\frac{1}{2} \arccos k_{21}, \eta_{20 l}=\frac{1}{2} \arccos k_{11}$. Here $k_{1 R}=-1, k_{1 /}=0.6$ 


\section{2 $\hat{P} \hat{T} \hat{C}$-invariant multi-soliton solutions of a nonlocal four-place DNLS equation} Replacing $t$ by $i t, x$ by $-i x$ and $q$ by $\frac{1}{2} q$ in the nonlocal four-place DNLS equation (12), it becomes

$$
\begin{aligned}
i q_{t} & +q_{x x}-\frac{1}{8}\left[q+q^{*}(x,-t)\right]^{2}\left[q(-x, t)+q^{*}(-x, t)\right]\left[q^{*}(-x, t) q^{*}(x,-t)-q q(-x,-t)\right] \\
& +\frac{i}{2} q\left[q^{*}(-x, t)+q(-x,-t)\right]\left[q^{*}(x,-t)+q\right]_{x} \\
& +\frac{i}{2}\left[\left(q+q^{*}(x,-t)\right)\left(q q(-x,-t)-q^{*}(x,-t) q^{*}(-x, t)\right)\right]_{x}=0 .
\end{aligned}
$$

In this section, we want to construct the soliton solutions of the nonlocal four-place DNLS equation (26). First, setting $q=q^{*}(x,-t)$ in Eq. (26), it reduces to

$$
i q_{t}+q_{x x}+2 i q\left[q^{*}(-x, t)\right] q_{x}=0
$$

Then setting $q(-x, t)=q$, Eq. (27) becomes the DNLS case (3). Thus, the two-soliton solution of the nonlocal four-place DNLS equation (26) can be obtained when we take $k_{2}=-k_{1}$ in Eq. (22),

$$
\begin{aligned}
& q=\left(| 2 k _ { 1 } | | k _ { 1 } + k _ { 1 } ^ { * } | \left[e^{i \eta_{1 I}} \cosh \left[\eta_{2 R}+i(\alpha-\beta)+\frac{1}{4}\left(\log k_{1}^{*}-\log k_{1}\right)\right]\right.\right.
\end{aligned}
$$

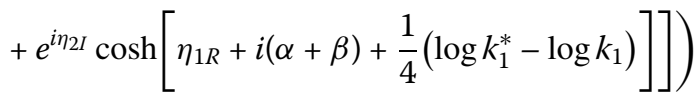

$$
\begin{aligned}
& /\left(\left|2 k_{1}\right|^{2} \cosh \left(\eta_{1 R}+\eta_{2 R}+\frac{1}{4} \log \frac{k_{1}^{2}}{k_{1}^{* 2}}\right)\right. \\
& \left.+\left|k_{1}+k_{1}^{*}\right|^{2} \cosh \left(\eta_{1 R}-\eta_{2 R}\right)+4 k_{1 I}^{2} \cos \left(\eta_{1 I}-\eta_{2 I}\right)\right) \text {, }
\end{aligned}
$$

with

$$
\begin{aligned}
& \eta_{1 R}=-k_{1 I} x+2 k_{1 R} k_{1 I} t, \quad \eta_{2 R}=k_{1 I} x+2 k_{1 R} k_{1 I} t, \\
& \eta_{1 I}=k_{1 R} x-\left(k_{1 R}^{2}-k_{1 I}^{2}\right) t+\eta_{10 I}, \quad \eta_{2 I}=-k_{1 R} x-\left(k_{1 R}^{2}-k_{1 I}^{2}\right) t+\eta_{20 I}, \\
& \eta_{10 I}=\eta_{20 I}=\frac{1}{2} \arccos \frac{\left(k_{1 I}^{2}-k_{1 R}^{2}\right)^{2}-4\left(k_{1 I} k_{1 R}\right)^{2}}{-4 k_{1 I}^{2}\left(k_{1 I}^{2}+k_{1 R}^{2}\right)^{\frac{3}{2}}},
\end{aligned}
$$

and

$$
\alpha=\operatorname{arctant} \frac{k_{1 I}}{k_{1 R}}, \quad \beta=0 .
$$

The interaction of the two-soliton solution (28) is illustrated in Fig. 3. The first column show the density plots of the two-soliton solution with $k_{1 R}=1, k_{1 I}=1, k_{1 R}=3, k_{1 I}=1$ and $k_{1 R}=1, k_{1 I}=3$, respectively. Pictures (a2)-(a4), (b2)-(b4) and (c2)-(c4) reveal the interaction process of the corresponding two-soliton for different choice of the real part and imparity part. Figure 3 reveals that the real part $k_{1 R}$ and imparity $k_{1 I}$ influence the interaction of the two-soliton solution. 


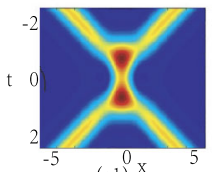

$(\text { a1 })^{x}$

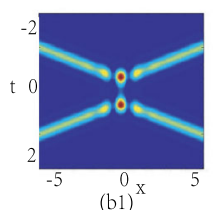

(b1)

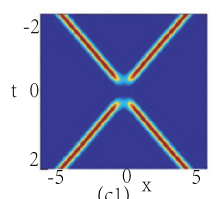

(c1) $\mathrm{x}$

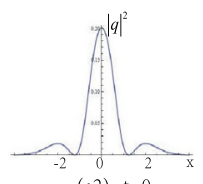

(a2) $t=0$
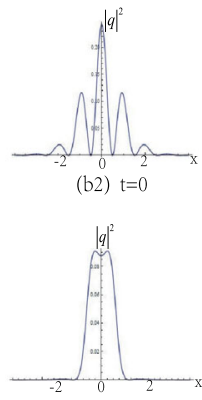

(c2) $\mathrm{t}=0$

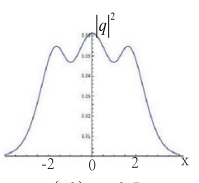

(a3) $\mathrm{t}=0.5$

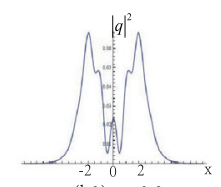

(b3) $\mathrm{t}=0.3$

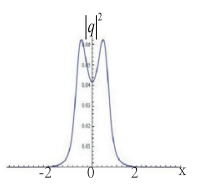

(c3) $\mathrm{t}=0.08$

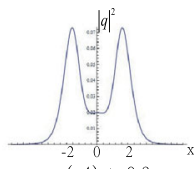

(a4) $\mathrm{t}=0.8$
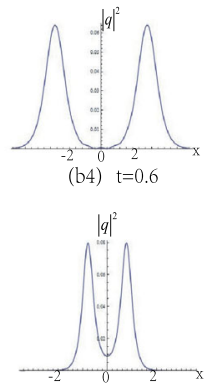

(c4) $\mathrm{t}=0.2$

Figure 3 Interaction of the two-soliton solution for the nonlocal four-place DNLS equation (26). Pictures (a1)-(a4) show the interaction of the two-soliton for $k_{1 R}=1, k_{1 /}=1$. Pictures (b1)-(b4) show the interaction of the two-soliton for $k_{1 R}=3, k_{1 /}=1$. Pictures (c1)-(c4) show the interaction of the two-soliton for $k_{1 R}=1, k_{1 /}=3$

\section{Conclusions}

Multi-place systems are important in both mathematical and physical fields. In this paper, we first construct the coupled CLL system and address its Lax pair which guarantees the integrability of the coupled CLL system. Then some kinds of nonlocal TDNLS equations and FDNLS equations are proposed by using the $\hat{P} \hat{T} \hat{C}$-symmetry.

$\hat{P} \hat{T} \hat{C}$-symmetry can be used not only to establish multi-place systems but also to solve the multi-place systems. With the help of the $\hat{P} \hat{T} \hat{C}$-symmetry, we not only obtain the onesoliton solution and periodic two-soliton solution of a nonlocal TDNLS equation but also work out the two-soliton solution of a nonlocal FDNLS equation for the first time. It is interesting to find that the arbitrary constant in the real part of $\eta_{j}$ can influence the interaction process of the two-soliton for the TDNLS equation and new dynamical behaviors are analyzed in Fig. 2. For the FDNLS equation, it is interesting to find that the real part $k_{j R}$ and the imparity $k_{j I}$ of the parameter $k_{j}$ influence the interaction process of the two-soliton and the dynamics as demonstrated in Fig. 3.

From the results of this paper, we find that there are some new interesting phenomena in the nonlocal multi-place systems. So it is significant to study the nonlocal multi-place systems.

Acknowledgements

The authors are very grateful to Professor S.Y. Lou for his guidance.

\section{Funding}

This work was supported by Beijing Natural Science Foundation (grand number 1182009) and NSFC under grants Nos. 11471182.

\section{Abbreviations}

KdV, Korteweg-de Vries; mKdV, modified Korteweg-de Vries; KP, Kadomtsev-Petviashvili; DS, Davey-Stewartson. 
Authors' contributions

The main ideal of this paper was proposed by YY. YY constructed the nonlocal-derivative NLS equation from the reduction of the coupled CLL system and carry out the computations and analysis for the group-invariant solutions. YH participated in calculations of the solutions and draw all the pictures. YY wrote the whole paper and YH revised the manuscript. All authors read and approved the final manuscript.

\section{Author details}

${ }^{1}$ Department of Applied Mathematics, China Agricultural University, Beijing, China. ${ }^{2}$ School of Mathematics and Physics, North China Electric Power University, Beijing, China.

\section{Publisher's Note}

Springer Nature remains neutral with regard to jurisdictional claims in published maps and institutional affiliations.

Received: 5 May 2019 Accepted: 23 January 2020 Published online: 19 March 2020

\section{References}

1. Lou, S.Y.: Alice-Bob Physics, $P_{s}-T_{d}-C$ principles and multi-soliton solutions (2016) arXiv:1603.03975V2 [nlin.SI]

2. Ablowitz, M.J., Musslimani, Z.H.: Integrable nonlocal nonlinear Schrödinger equation. Phys. Rev. Lett. 110, 064105 (2013)

3. Gadzhimuradov, T.A., Agalarov, A.M.: Towards a gauge-equivalent magnetic structure of the nonlocal nonlinear Schrodinger equation. Phys. Rev. A 93, 062124 (2016)

4. Jia, M., Lou, S.Y.: Exact PSTd invariant and PSTd symmetric breaking solutions, symmetry reductions and Backlund transformations for an AB-KdV system. Phys. Lett. A 382, 1157-1166 (2018)

5. Lou, S.Y.: Prohibition caused by nonlocality for nonlocal Boussinesq-KdV type systems. Stud. Appl. Math. 2019, 1-16 (2019)

6. Li, C., Lou, S.Y., Jia, M.: Coherent structure of Alice-Bob modified Korteweg-de Vries equation. Nonlinear Dyn. 93, 1799-1808 (2018)

7. Ablowitz, M.J., Musslimani, Z.H.: Inverse scattering transform for the integrable nonlocal nonlinear Schrodinger equation. Nonlinearity 29, 915-946 (2016)

8. Ji, J.L., Zhu, Z.N.: Soliton solutions of an integrable nonlocal modified Korteweg-de Vries equation through inverse scattering transform. J. Math. Anal. Appl. 453, 973-984 (2017)

9. Ablowitz, M.J., Musslimani, Z.H.: Integrable discrete PT symmetric model. Phys. Rev. E 90, 032912 (2014)

10. Lou, S.Y.: Alice-Bob systems, $\widehat{P T C}$ symmetry invariant and symmetry breaking soliton solutions. J. Math. Phys. 59, 083507 (2018)

11. Huang, L.L., Chen, Y.: Nonlocal symmetry and similarity reductions for the Drinfeld-Sokolov-Satsuma-Hirota system. Appl. Math. Lett. 64, 177-184 (2017)

12. Yang, B., Chen, Y.: Dynamics of rogue waves in the partially PT-symmetric nonlocal Davey-Stewartson systems. Commun. Nonlinear Sci. Numer. Simul. 69, 287-303 (2019)

13. Fokas, A.S.: Integrable multidimensional versions of the nonlocal nonlinear Schrodinger equation. Nonlinearity 29 319-324 (2016)

14. Rao, J., Cheng, Y., He, J.S.: Rational and semirational solutions of the nonlocal Davey-Stewartson equations. Stud. Appl. Math. 139, 568-598 (2017)

15. Sinha, D., Ghosh, P.K.: Integrable nonlocal vector nonlinear Schrodinger equation with self-induced parity-time-symmetric potential. Phys. Lett. A 23, 124-128 (2017)

16. Ren, B.: Interaction solutions for mKP equation with nonlocal symmetry reductions and CTE method. Phys. Scr. 90 $065206(2015)$

17. Chen, J.C., Xin, X.P., Chen, Y.: A direct algorithm of one-dimensional optimal system for the group invariant solutions. J. Math. Phys. 55, 053508 (2014)

18. Chen, Z.G., Segev, M., Christodoulides, D.N.: Optical spatial solitons: historical overview and recent advances. Rep. Prog. Phys. 75, 086401 (2012)

19. Lederer, F., Stegeman, D.I., et al.: Discrete solitons in optics. Phys. Rep. 463, 112-126 (2008)

20. Yang, J.: Nonlinear waves in integrable and non integrable systems. SIAM Mathematical Modeling and Computation (2010)

21. Wang, M.L., Zhou, Y.B., Li, Z.B.: Application of a homogeneous balance method to exact solutions of nonlinear equations in mathematical physics. Phys. Lett. A 216, 67-75 (1996)

22. Baleanu, D., Inc, M., Yusuf, A., Aliyu, A.l.: Traveling wave solutions and conservation laws for nonlinear evolution equation. J. Math. Phys. 59, 023506 (2018)

23. Ghanbari, B., Yusuf, A., Inc, M., Baleanu, D.: The new exact solitary wave solutions and stability analysis for the $(2+1)$-dimensional Zakharov-Kuznetsov equation. Adv. Differ. Equ. 2019, 49 (2019)

24. Inc, M., Aliyu, A.I., Yusuf, A., Baleanu, D.: Optical solitons and modulation instability analysis to the quadratic-cubic nonlinear Schrodinger equation. Nonlinear Anal., Model. Control 24, 20-33 (2019)

25. Inc, M., Aliyu, A.I., Yusuf, A., Baleanu, D.: Dark-bright optical solitary waves and modulation instability analysis with $(2+1)$-dimensional cubic-quintic nonlinear Schrodinger equation. Waves Random Complex Media 29, 393-402 (2019)

26. Ruzhansky, M., Cho, Y.J., Agarwal, P., Area, I.: Advances in Real and Complex Analysis with Applications. Springer, Singapore (2017)

27. Agarwal, P., Deniz, S., Jain, S., Alderremy, A.A., Shaban, A: A new analysis of a partial differential equation arising in biology and population genetics via semi analytical techniques. Phys. A, Stat. Mech. Appl. 2019, 122769 (2019)

28. Saoudi, K., Agarwal, P., Kumam, P., Ghanmi, A., Thounthong, P.: The Nehari manifold for a boundary value problem involving Riemann-Liouville fractional derivative. Adv. Differ. Equ. 2018, 263 (2018) 
29. Agarwal, P., Choi, J., Paris, R.B.: Extended Riemann-Liouville fractional derivative operator and its applications. J. Nonlinear Sci. Appl. 8, 451-466 (2015)

30. Srivastava, H.M., Agarwal, P.: Certain fractional integral operators and the generalized incomplete hypergeometric functions. Appl. Appl. Math. 8, 333-345 (2013)

31. Agarwal, P.: Further results on fractional calculus of Saigo operators. Appl. Appl. Math. 7, 585-594 (2012)

32. Agarwal, P.: Some inequalities involving Hadamardtype kfractional integral operators. Math. Methods Appl. Sci. 40 3882-3891 (2017)

33. Sato, M.: Soliton equations as dynamical systems on infinite dimensional Grassmann manifold. RIMS Kyoto Univ. Kokyuroku 439, 30 (1981)

34. Gilson, C., Hietarinta, J., Nimmo, J., Ohta, Y.: Sasa-Satsuma higher-order nonlinear Schrödinger equation and its bilinearization and multisoliton solutions. Phys. Rev. E 68, 016614 (2003)

35. Mao, H., Liu, Q.P.: Backlund-Darboux transformations and discretizations of $N=2 a=-2$ supersymmetric KdV equation. Phys. Lett. A 382, 253-258 (2018)

36. Amjad, Z., Haider, B.: Binary Darboux transformations of the supersymmetric Heisenberg magnet model. Theor. Math Phys. 199, 784-797 (2019)

37. Cheng, X.P., Lou, S.Y., Chen, C.L., Tang, X.Y.: Interactions between solitons and other nonlinear Schrodinger waves. Phys. Rev. E 89, 043202 (2014)

38. Lou, S.Y., Hu, X.R., Chen, Y.: Nonlocal symmetries related to Backlund transformation and their applications. J. Phys. A, Math. Theor. 45, 155209 (2012)

39. Xiao, Y., Fan, E.G.: A Riemann-Hilbert Approach to the Harry-Dym Equation on the Line. Chin. Ann. Math., Ser. B 37, 373-384 (2016)

40. Zhu, Q.Z., Xu, J., Fan, E.G.: The Riemann-Hilbert problem and long-time asymptotics for the Kundu-Eckhaus equation with decaying initial value. Appl. Math. Lett. 76, 81-89 (2018)

41. Yang, X.J., Tenreiro Machado, J.A.: A new fractal nonlinear Burgers' equation arising in the acoustic signals propagation. Math. Methods Appl. Sci. 42(18), 7539-7544 (2019). https://doi.org/10.1002/mma.5904

42. Yang, X.J., Gao, F., Srivastava, H.M.: A new computational approach for solving nonlinear local fractional PDEs. J. Comput. Appl. Math. 339, 285-296 (2018)

43. Gao, F., Yang, X.J., Ju, Y.: Exact traveling-wave solutions for one-dimensional modified Korteweg-de Vries equation defined on Cantor sets. Fractals 27, 1940010 (2019)

44. Yang, X.J., Tenreiro Machado, J.A., Baleanu, D.: Exact traveling-wave solution for local fractional Boussinesq equation in fractal domain. Fractals 25, 1740006 (2017)

45. Yang, X.J., Gao, F., Srivastava, H.M.: Exact travelling wave solutions for the local fractional two-dimensional Burgers-type equations. Comput. Math. Appl. 73, 203-210 (2017)

46. Yang, X.J., Tenreiro Machado, J.A., Baleanu, D., Cattani, C.: On exact traveling-wave solutions for local fractional Korteweg-de Vries equation. Chaos, Interdiscip. J. Nonlinear Sci. 26, 084312 (2016)

47. Gao, F., Yang, X.J., Zhang, Y.F.: Exact traveling wave solutions for a new non-linear heat transfer equation. Therm. Sci. 21, 1833-1838 (2017)

48. Gao, F., Yang, X.J., Srivastava, H.M.: Exact traveling-wave solutions for linear and non-linear heat transfer equations. Therm. Sci. 21, 2307-2311 (2017)

49. Sarfraz, H., Saleem, U.: Darboux transformation and multi-soliton solutions of local/nonlocal N-wave interactions. Mod. Phys. Lett. A 32, 1750196 (2017)

50. Ji, J.L., Zhu, Z.N.: On a nonlocal modified Korteweg-de Vries equation: integrability, Darboux transformation and soliton solutions. Commun. Nonlinear Sci. Numer. Simul. 42,699-708 (2017)

51. Li, M., Xu, T.: Dark and antidark soliton interactions in the nonlocal nonlinear Schrodinger equation with the self-induced parity-time-symmetric potential. Phys. Rev. E 91, 033202 (2015)

52. Xu, T., Li, H., Zhang, H., Li, M., Lan, S.: Darboux transformation and analytic solutions of the discrete PT-symmetric nonlocal nonlinear Schrodinger equation. Appl. Math. Lett. 63, 88-94 (2017)

53. Chen, K., Zhang, D.J.: Solutions of the nonlocal nonlinear Schrodinger hierarchy via reduction. Appl. Math. Lett. 75 $82-88(2018)$

54. Xu, Z.X., Chow, K.W.: Breathers and rogue waves for a third order nonlocal partial differential equation by a bilinear transformation. Appl. Math. Lett. 56, 72-77 (2016)

55. Chen, K., Deng, X., Lou, S.Y., Zhang, D.J.: Solutions of nonlocal equations reduced from the AKNS hierarchy. Stud. Appl. Math. 113,113-141 (2018)

56. Loris, I., Willox, R.: Soliton solutions of Wronskian form to the nonlocal Boussinesq equation. J. Phys. Soc. Jpn. 65 , 383-388 (1996)

57. Liu, Y., Mihalache, D., He, J.S.: Families of rational solutions of the $y$-nonlocal Davey-Stewartson II equation. Nonlinear Dyn. 90, 2445-2455 (2017)

58. Zhai, W., Chen, D.Y.: N-soliton solutions of general nonlinear Schrodinger equation with derivative. Commun. Theor. Phys. 49, 1101-1104 (2008)

59. Chen, H.H., Lee, Y.C., Liu, C.S.: Integrability of non-linear Hamiltonian systems by inverse scattering method. Phys. Scr. 20, 490-492 (1979) 